\title{
Patrones de distribución de los parámetros físicos y metales pesados en la cuenca media y baja del río
} Utcubamba

\section{Distribution patterns of physical parameters and heavy metals in the middle and lower Utcubamba river basin}

\author{
Jesús Rascón ${ }^{1 *}(\mathbb{D})$, Dámaris Leiva-Tafur ${ }^{1} \mathbb{D}$, Lily del Pilar Juárez Contreras ${ }^{1} \mathbb{D}$, Elder Chichipe Vela ${ }^{1}$, Carlos Santa Cruz
}

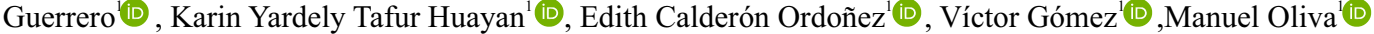

\section{RESUMEN}

El río Utcubamba es uno de los principales cuerpos de agua dulce en el Departamento de Amazonas. A lo largo de su cauce se encuentra expuesto a una gran cantidad de actividades antropogénicas que ocasionan el deterioro de su ecosistema. La escasez de información sobre el transporte de elementos químicos por este río, y su posterior descarga en el río Utcubamba, impiden elaborar planes adecuados para el uso sustentable de sus recursos y así tratar de mitigar el impacto generado por las actividades humanas. La presente investigación tuvo como objetivos determinar la variación espacial, del río Utcubamba a lo largo de las cuencas media y baja e identificar el comportamiento de los contaminantes, así como su distribución. Se seleccionaron once estaciones de muestreo, seis en la cuenca media y cinco en la cuenca baja. En general, el transporte medio de metales pesados aumentó hacia la cuenca baja, debido principalmente a que ambas cuencas, se encuentran impactadas por la acción antropogénica.

Palabras clave: variación espacial, Utcubamba, Amazonas.

\begin{abstract}
The Utcubamba River is one of the main bodies of fresh water in the Department of Amazonas. Throughout its course it is exposed to a large number of anthropogenic activities that cause the deterioration of its ecosystem. The lack of information on the transport of chemical elements through this river, y its subsequent discharge into the Utcubamba River, prevent the development of adequate plans for the sustainable use of its resources and thus try to mitigate the impact generated by human activities. The purpose of this research was to determine the spatial variation of the Utcubamba River along the middle and lower basins and identify the behavior of the pollutants, as well as their distribution. Eleven sampling stations were selected, six in the middle basin and five in the lower basin. In general, the average transport of heavy metals increased towards the lower basin, mainly due to the fact that both basins are impacted by the anthropogenic action.
\end{abstract}

Keywords: spatial variation, Utcubamba, Amazonas.

\footnotetext{
${ }^{1}$ Universidad Nacional Toribio Rodríguez de Mendoza de Amazonas, Instituto de Investigación para el Desarrollo Sustentable de Ceja de Selva, Chachapoyas, Perú

*Autor de correspondencia. E-mail: jesus.rascon@untrm.edu.pe
} 


\section{INTRODUCCIÓN}

El río Utcubamba posee una longitud aproximada de $250 \mathrm{~km}$ desde su nacimiento en el caserío Atuen (provincia Chachapoyas), hasta su desembocadura en el rio Marañon, siendo su principal tributario (Gamarra et al., 2018b). Atraviesa de forma vertical, todo el Departamento de Amazonas desde el sur hasta el norte, pasando por las provincias de Chachapoyas, Bagua, Bongará, Luya y Utcubamba. Se caracteriza por ser unos de los ríos más caudalosos del norte de Perú, sino se tiene en cuenta al Marañón y al Amazonas (Maicelo et al., 2014). El régimen pluviométrico de la cuenca del río Utcubamba, se caracteriza por una época húmeda que comienza a partir del mes de diciembre y finaliza en junio. Por otra parte, la época seca comienza en el mes de julio y finaliza en noviembre teniendo sus máximos valores en el bimestre agosto-septiembre (EfusFernández y Pinedo-Trigoso, 2019, Rascon et al., 2020; 2021a). En la cuenca media se desarrollan actividades agrícolas y ganaderas, dónde se localizan poblaciones como la ciudad de Chachapoyas y Pedro Ruiz (Corroto et al., 2018). La cuenca baja es la de mayor población, localizándose la principal ciudad del Departamento, Bagua Grandes, además de otra ciudad grande como Bagua. Allí esencialmente se desarrollan actividades agrícolas, relacionadas con el arroz. Las aguas servidas provenientes de todas estas actividades humanas son descargadas en el cauce del río, sin ningún tipo de tratamiento. Estas llevan consigo una elevada concentración de elementos químicos, alterando la calidad ambiental del río, así como también de la zona costera donde este desemboca, impidiendo así su uso y aprovechamiento humano (Gamarra et al., 2018a).

A pesar de lo expuesto, es escasa la información sobre el volumen de metales pesados que son descargados hacia el río Utcubamba. No hay investigaciones sobre los patrones de distribución en la cuenca media y baja. Por ello, es necesario estimar estos patrones de distribución de los metales pesados y los parámetros físicos más relacionados con ellos, para buscar alternativas orientadas a mitigar el impacto generado por las actividades humanas en el ecosistema fluvial, ofreciendo una oportunidad para mejorar el aprovechamiento del recurso hídrico y el manejo de la zona costera. En total se estudiaron 11 metales pesados y 7 parámetros ambientales.

\section{MATERIAL Y MÉTODOS}

\section{Área de estudio}

La cuenca media y baja del río Utcubamba se encuentra atravesando varias provincias, del Departamento Amazonas, entre ellas Bongará, Luya, Utcubamba y Bagua. El río Utcubamba tiene una longitud de $250 \mathrm{~km}$ (Figura 1). Nace en los Andes, en la provincia de Chachapoyas y desemboca en el río Marañón en la provincia de Bagua, siendo uno de sus principales tributarios (Barboza et al., 2017). Pertenece a la Región Hidrográfica del Amazonas. Las cuencas media y baja, se sitúan desde una altitud de 1686 m.s.n.m hasta los 360 m.s.n.m, comprendida dentro del bosque tropical estacionalmente seco y muy seco (Linares-Palomino, 2004; Gamarra et al. 2018c).

Las actividades más representativas en la zona, son la actividad ganadera y la actividad agrícola. En la cuenca media, destacan los predominios con cultivos andinos como la papa (Solanum tuberosum L.), el camote (Ipomoea batatas (L.) Lam.) o el maíz (Zea mays L.) entre otros (Tapia y Fries, 2007; Corroto et al., 2018). Mientras que, en la cuenca baja, destacan los predominios con cultivos de arroz (Oryza latifolia Desv.) y la agricultura de subsistencia (Corroto et al., 2018).

\section{Metodología empleada}

Los muestreos se realizaron en el mes de Agosto de 2018, en época seca. Se establecieron 11 puntos de muestro a lo largo del río Utcubamba siguiendo la metodología de Gamarra et al. (2018a). Las estaciones de la 1 a la 6 corresponden a la cuenca medía, mientras que las estaciones de la 7 a la 11 corresponden a la cuenca baja. Los puntos se establecieron previamente en fase de gabinete con la ayuda del software informático ArcGIS y confirmados en campo con un receptor de Posicionamiento Global (GPS), marca Garmin, modelo Oregon 650 (Figura 1).

La recolección, almacenamiento y traslado de las mues- 


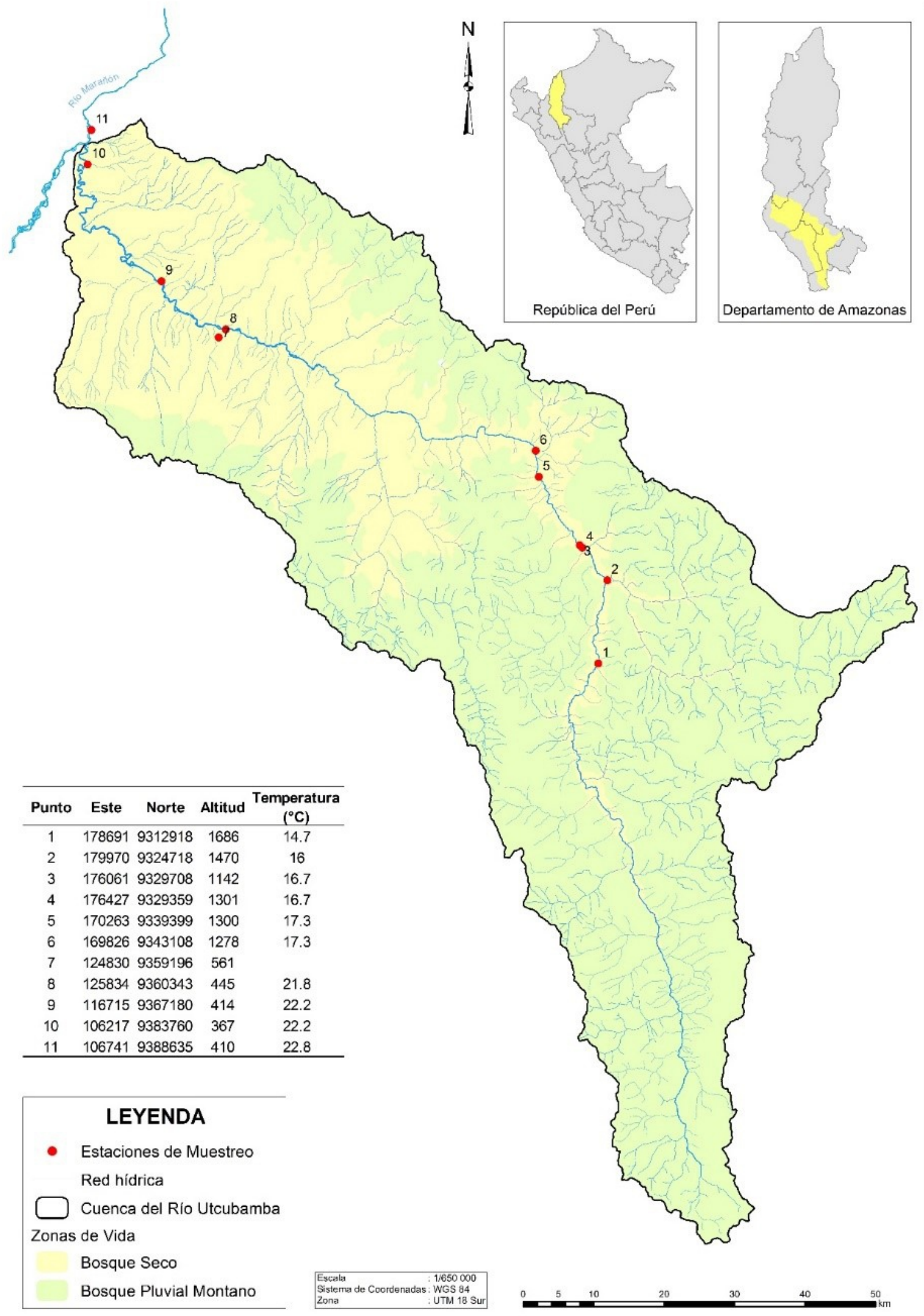

Figura 1. Ubicación de las estaciones de muestreo en la Cuenca Baja y Media del río Utcubamba. 
tras y los análisis de laboratorio, se realizaron de acuerdo con APHA, AWWA, y WEF (2017). Se realizaron tres repeticiones en cada estación de muestreo. Los parámetros físicos estudiados fueron $\mathrm{pH}(\mathrm{pH}, 1: 1$; Método 4500-H+), temperatura (Temp, ${ }^{\circ} \mathrm{C}$; Método 2550B), oxígeno disuelto (OD, $\mathrm{O}_{2} \mathrm{mg} / \mathrm{L}$; Método 4500$\mathrm{O} \mathrm{G})$, conductividad eléctrica ( $\mathrm{CE}, \mu \mathrm{S} / \mathrm{cm}$; Método 2510 B), sólidos disueltos totales (SDT, mg/L; Método $2510 \mathrm{~B}$ ) fueron medidos con un equipo multiparamétrico marca SI Analytics, modelo HandyLab 680, turbidez (Turbi, UNT; Método 180.1 EPA) fue medido con un equipo turbidímetro marca hach modelo $2100 \mathrm{q}$, y sólidos totales (ST, mg/L; Método 2540 B) fueron medidos usando una balanza marca Pioneer, modelo PX232 y una estufa marca MMM Group, modelo ECOCELL. En cuanto a los metales estudiados, fueron Magnesio (Mg mg/L), Aluminio (Al mg/L), Manganeso ( $\mathrm{Mn}$ $\mathrm{mg} / \mathrm{L}$ ), Barios (Ba mg/L), Litio (Li mg/L), Hierro (Fe mg/L), Zinc (Zn mg/L), Cobre (Cu mg/L), Cobalto (Co $\mathrm{mg} / \mathrm{L})$, Cadmio (Cd mg/L) y Plomo (Pb mg/L). Todos ellos fueron medidos usando el Método 3120-B, con un espectrofotómetro de emisión atómica, marca Agilent Technologies, modelo 4210 MP-AES

Para el análisis de datos, se realizó un gráfico de boxplot para cada parámetro, junto a la estadística descriptiva de los parámetros, y una correlación de Spearman, para comprobar cómo se relación los metales pesados y los parámetros ambientales entre ellos. Posteriormente, se calculó el promedio de cada estación y se graficó para ver la evolución espacial de cada parámetro a lo largo de la cuenca media y baja. Todos los análisis estadísticos se realizaron a un nivel de significancia de $\mathrm{p}<0,05$ con el software estadístico R (R Core Team, 2019).

\section{RESULTADOS}

\section{Relación de los parámetros con las estaciones}

La variación espacial tuvo un impacto significativo en la distribución de todos los parámetros fisicoquímicos, mientras que dentro de los metales pesados tanto el Mn como el $\mathrm{Cd}$, no tiene ninguna diferencia significativa entre las estaciones de muestreo. Cabe mencionar que como los valores de $\mathrm{Al}$ y Co fueron 0 , no se tienen en cuenta para este análisis (Tabla 1).

\section{Patrones de distribución}

La distribución espacial de los parámetros, según las estaciones de muestreo, arroja cuatro grupos bien definidos. Un grupo principal donde se encuentran la mayoría de parámetros, $\mathrm{y}$ tres grupos con un solo parámetros (Mn, Li y Zn) (Figura 2)

La distribución espacial de estaciones, según los parámetros muestreados, arroja cuatro grupos bien definidos. Un grupo principal donde se encuentran las estaciones de la cuenca baja EM8, EM9, EM10 y EM11, que son las que presentan más contaminantes por metales pesados. Luego tenemos un grupo con EM3, EM6 (Cuenca media) y EM7 (Cuenca baja), lo que indica que, hasta la EM7, no hay diferencias por contaminación. Luego tenemos un grupo con tres estaciones de la cuenca media (EM2, EM4 y EM5) y un último grupo donde solo esta EM1 (Figura 3).

\section{DISCUSIÓN}

El patrón identificado en el presente estudio en cuanto a la variación espacial del caudal fue el típico para estos sistemas fluviales en los cuales se observa un incremento desde la cuenca alta hacia la cuenca baja (OrtizArenas et al., 2017). El aporte de varios nutrientes,

Tabla 1. Prueba de Kruskal Wallis entre los parámetros ambientales y metales pesados y las estaciones de muestreo

\begin{tabular}{|c|c|c|c|c|c|c|c|c|}
\hline Parámetros & $\mathbf{F}$ & p-valor & Parámetros & $\mathbf{F}$ & p-valor & Parámetros & $\mathbf{F}$ & p-valor \\
\hline pH (1:1) & 27.51 & $0.0021^{*}$ & ST (mg/L) & 31.77 & $0,0004^{*}$ & Fe $(\mathrm{mg} / \mathrm{L})$ & 26.11 & $0,0036^{*}$ \\
\hline $\mathbf{T}\left({ }^{\circ} \mathrm{C}\right)$ & 30.36 & $0,0007^{*}$ & $\operatorname{Mg}(\mathrm{mg} / \mathrm{L})$ & 31.67 & $0,0005^{*}$ & $\mathrm{Zn}(\mathrm{mg} / \mathrm{L})$ & 27.69 & $0,0020^{*}$ \\
\hline Turbidez (UNT) & 30.34 & $0,0008^{*}$ & $\mathrm{Al}(\mathrm{mg} / \mathrm{L})$ & 0 & 0 & $\mathrm{Cu}(\mathrm{mg} / \mathrm{L})$ & 32.00 & $0,0004^{*}$ \\
\hline OD (mg/L) & 30.98 & $0,0006^{*}$ & $\operatorname{Mn}(\mathrm{mg} / \mathrm{L})$ & 10.14 & 0,4285 n.s. & Co $(\mathrm{mg} / \mathrm{L})$ & 0 & 0 \\
\hline $\mathrm{CE}(\boldsymbol{\mu S} / \mathbf{c m})$ & 31.82 & $0.0004^{*}$ & Ba $(\mathrm{mg} / \mathrm{L})$ & 24.31 & $0,0068 *$ & Cd $(\mathrm{mg} / \mathrm{L})$ & 5.95 & 0.8190n.s. \\
\hline SDT (mg/L) & 31.67 & $0,0005^{*}$ & Li (mg/L) & 31.63 & $0,0005 *$ & Pb (mg/L) & 23.83 & $0,0081^{*}$ \\
\hline
\end{tabular}




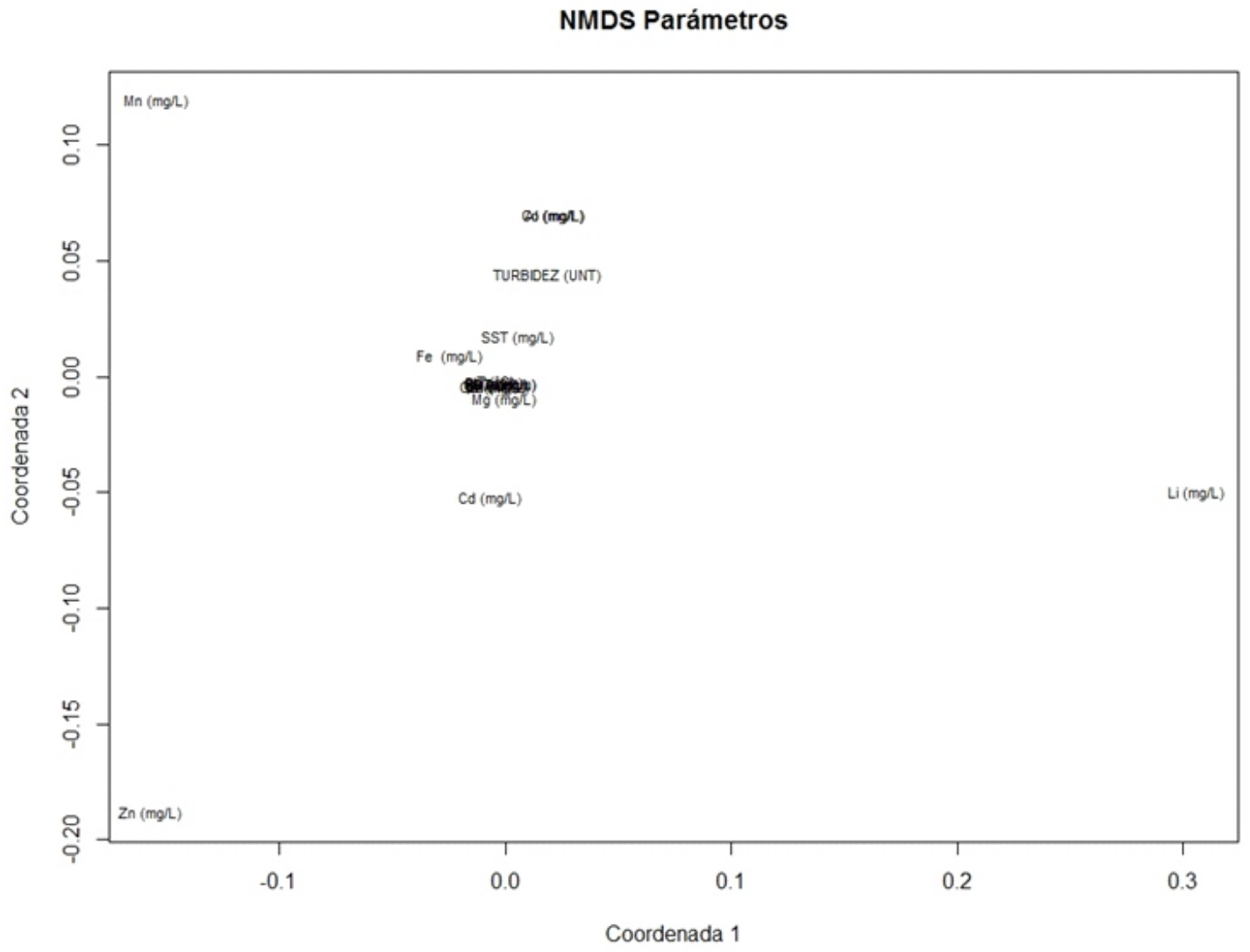

Figura 2. Ordenamiento de los 18 parámetros muestreados en función de la variación espacial.

NMDS ESTACIONES

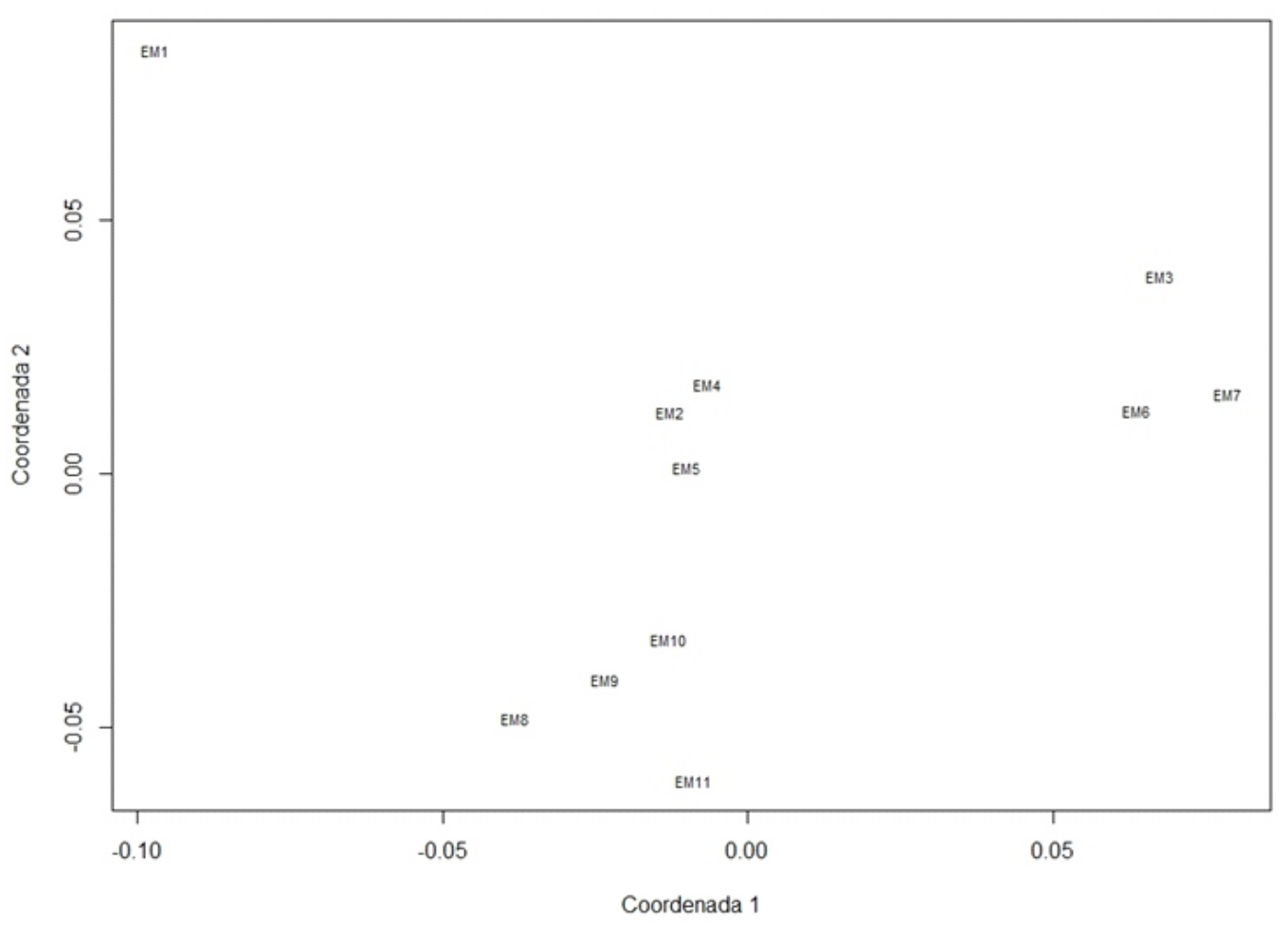

Figura 3. Ordenamiento de las 11 estaciones de muestreo en función de los parámetros ambientales y metales pesados. 
relacionados con la agricultura y a la ganadería se dan desde la cuenca media hasta la baja. El comportamiento temporal del caudal del rio Utcubamba depende significativamente de las precipitaciones ocurridas en la cuenca, es por esto que los máximos valores del caudal corresponden a aquellos meses con mayores precipitaciones (Gao et al., 2014).

La cuenca alta del río Utcubamba se caracteriza por estar en la sierra del Calla-Calla, con pueblos que en la mayoría de los casos no poseen sistemas de tratamiento de agua. En esta zona, además, se realizan pequeñas actividades agrícolas y de cría de animales, que descargan sus desechos directamente al río. En las actividades agrícolas de la cuenca se emplean fertilizantes como abono de la tierra, pudiendo representar ésta una de las principales fuentes de compuestos nitrogenados, que ingresan a través de las escorrentías (Ogbozige y Alfa, 2019; Khan et al., 2017; Li et al., 2015). Varios autores han observado una situación similar para especies nitrogenadas en el río Orituco, señalando que el aumento en las concentraciones de nitrato estaba relacionado con las estaciones del río que históricamente habían sido perturbadas por el uso de fertilizantes (Cressa, 1993; Flores et al., 2017). La cuenca media es una zona agrícola, principalmente para cultivos andinos. Al mismo, cuenta con una gran actividad ganadera (Corroto et al., 2018). Todas estas actividades incrementan la descarga de compuestos nutritivos que viene desde la cuenca alta. A esto hay que añadir, las descargas de la ciudad de Chachapoyas, la cual tiene dos PTAR, pero no se encuentran en funcionamiento, así como de las pequeñas poblaciones en las márgenes del río no poseen este tipo de redes de recolección de aguas servidas, lo que trae como consecuencia que las comunidades viertan sus desechos o al menos parte de ellos en los canales pluviales aun cuando posean pozos sépticos (Gamarra et al., 2018b). En la cuenca baja la situación es bastante grave, ya que el río Utcubamba cruza la ciudad de Bagua Grande para desembocar sus aguas en el río Marañón. A lo largo de toda la cuenca baja, el río recibe las descargas de todos los cultivos de arroz, que hay en la zona, al ser una zona de gran producción arro- cera (Zamora-Huamán y Mejía-Risco, 2019). Al mismo tiempo, la ciudad de Bagua Grande, la cual es la más grande del Departamento de Amazonas también realiza descargas de elementos que provienen de las actividades humanas, aumentando el transporte de estos hacia el rio Marañón, algo normal en poblaciones de este departamento (Rascón et al. 2021b).

Respecto a la influencia de los metales pesados dentro del río Utcubamba, se ha evidenciado que la descarga de estos, según vamos avanzando por el río, vemos que van amentando, por eso se van agrupando las estaciones según las cuencas, además de que todos los parámetros influyen en el estado en el que se encuentran ambas cuencas, como vemos en la figura 3 y 4 . Resultados parecidos se han observado en el río Córdoba en Magdalena, Colombia. En dicho río, existe una alta uniformidad espacial del río en los sitios de muestreo, donde evidenciaron que la variación temporal reflejó de mejor manera los cambios en la calidad del agua del río Córdoba en su parte baja (Fontalvo et al., 2018).

\section{CONCLUSIONES}

Se demuestra que los parámetros se comportan de forma distinta según avanzan por el río Utcubamba, excepto para el $\mathrm{Al}$ y Co, que no presentaron valor alguno y para los valores de $\mathrm{Cd}$ y $\mathrm{Mn}$, lo que significa que la contaminación por estos metales, es constante a lo largo de las dos cuencas

La cuenca media y alta, tiene cuatro patrones de distribución, que serían, cuenca media-alta con poca contaminación, cuenca media-baja con indicios de contaminación, cuenca baja-alta con cierta contaminación y cuenca baja-baja, con gran contaminación. Estos contaminantes son sobre todo $\mathrm{Cd}, \mathrm{Mn}$ y $\mathrm{Pb}$.

Es necesario desarrollar una evaluación exhaustiva en el recorrido del río Utcubamba, para profundizar en la evaluación de Cadmio y Plomo, ya que la presencia de altas concentraciones de estos metales en el ser humano tiende a causar alteraciones en el sistema nervioso central y digestivo. 


\section{CONTRIBUCIÓN DE LOS AUTORES}

Todos los autores participaron en la conceptualización, metodología, investigación, redacción del manuscrito inicial, revisión bibliográfica, y en la revisión y aprobación del manuscrito final.

\section{CONFLICTO DE INTERESES}

Los autores declaran no tener conflicto de intereses.

\section{REFERENCIAS BIBLIOGRÁFICAS}

APHA, AWWA, y WEF. 2017. Standard Methods for the Examination of Water and Wastewater. Washington D.C. (EEUU): American Public Health Association/American Water Works Association/Water Environment Federation.

Barboza, E., F. Corroto, R. Salas, O. Gamarra, y D. Ballarín. 2017. "Hidrogeomorfología En Áreas Tropicales: Aplicación Del Índice Hidrogeomorfológico (IHG) En El Río Utcubamba (Perú)." Ecología Aplicada 16 (1). 3947.

Corroto, F., O. Gamarra, y E. Barboza. 2018. "Multivariate Assessment of Water Quality in the Utcubamba Basin (Peru)." Tecnologia $y$ Ciencias Del Agua 9 (5): 33-51. DOI:10.24850/j-tyca-2018-05-02.

Cressa, C. 1993. "Diel Variations in Some Chemical Variables of the Orituco River, Venezuela." Ecotropicos 6(2): 30-38.

Efus-Fernández, F. A., y R. Pinedo-Trigoso. 2019. "Estudio de Pavimentación En El AA.HH. Pedro Castro Alva, Distrito de Chachapoyas, Provicnia de Chachapoyas - Amazonas, Perú.” Tesis de Grado. Universidad Nacional Pedro Ruiz Gallo. Lambayaque (Perú).

Flores, M., M. Bermúdez, X. Bustos, M. Bernet, C. Viana, S. Arcia, y M. Schmitz. 2017. “Análisis de Los Procesos Controladores En La Evolución Topográfica de La Subcuenca Del Río Guárico, Venezuela: Mediante Métodos Multiherramientas." Terra Nueva Etapa 33 (54): 71-106.
Fontalvo, P. P., G. Orozco Berdugo, y J. Narváez Barandica. 2018. "Diversidad y Estructura Genética Del Prochilodus Magdalenae (Pisces: Prochilodontidae) Aguas Arriba y Abajo de La Represa Betania, Colombia." Intropica 9: 87. DOI:10.21676/23897864.2505.

Gamarra, O. A., M. A. Barrena-Gurbillón, E. Barboza-Castillo, J. Rascón, F. Corroto, y L. A. Taramona-Ruiz. 2018a. "Fuentes de Contaminación Estacionales En La Cuenca Del Río Utcubamba , Región Amazonas , Perú.” Arnaldoa 25 (1): 179-94. DOI: 10.22497/arnaldoa.251.25111

Gamarra, O. A., F. Corroto, M. A. Barrena-Gurbillón, J. Rascón, y J. Chávez-Ortiz. 2018b. Calidad Ecológica del Agua en la Cuenca del Río Utcubamba, Amazonas, Perú. Chachapoyas (Perú): Fondo Editorial UNTRM.

Gamarra, O. A., M. A. Barrena-Gurbillón, C. Ordinola, E. Barboza, D. Leiva, J. Rascón, F. Corroto, y L. A. Taramona. 2018c. "Calidad del Bosque de Ribera en la Cuenca del Río Utcubamba, Amazonas, Perú." Arnaldoa 25 (2): 653-78. DOI:10.22497/arnaldoa.252.25218.

Gao, Y., J. M. O'Neil, D. K. Stoecker, y J. C. Cornwell. 2014. "Photosynthesis and Nitrogen Fixation during Cyanobacteria Blooms in an Oligohaline and Tidal Freshwater Estuary." Aquatic Microbial Ecology 72 (2): 127-42. DOI:10.3354/ame01692.

Khan, M. Y.A., K. Muzamil Gani, y G. Joseph Chakrapani. 2017. "Spatial and Temporal Variations of Physicochemical and Heavy Metal Pollution in Ramganga River - a Tributary of River Ganges, India." Environmental Earth Sciences 76 (5). DOI:10.1007/s12665-0176547-3.

Li, X., T. Huang, W. Ma, X. Sun, y H. Zhang. 2015. "Effects of Rainfall Patterns on Water Quality in a Stratified Reservoir Subject to Eutrophication: Implications for Management." Science of the Total Environment 
$521-522$ :

$27-36$.

DOI:10.1016/j.scitotenv.2015.03.062.

Linares-Palomino, R. 2004. "Los Bosques Tropicales

Estacionalmente Secos : II . Fitogeografía y Composición Florística.” Arnaldoa 11 (1): 103-38.

Maicelo, J., K. Miñano, W. Bardales, y S. Leiva. 2014. "Inventario de Macroinvertebrados Bentónicos En La Cuenca Del Río Utcubamba, Región Amazonas (Perú).” Revista Indes 2 (2): 39-48. DOI:10.25127/indes.20140.

Ogbozige, F. J. y M. I. Alfa. 2019. "Land Use-Land Cover Effects on Surface Flowing Water Quality: A Statistical Approach.” Nigerian Journal of Technological Development 16(1): 25. DOI:10.4314/njtd.v16il.4.

Ortiz-Arenas, A. L., M. Ruiz-Ochoa, y J. P. RodríguezMiranda. 2017. "Planificación y Gestión de Los Recursos Hídricos : Una Revisión de La Importancia de La Variabilidad Climática." Revista Logos Ciencia \& Tecnologia 9 (1): 100-105.

Rascón, J., W. Gosgot Angeles, M. Oliva, L. Quiñones, y M. Á. Barrena Gurbillón. 2020. "Determinación de Las Épocas Lluviosas y Secas En La Ciudad de Chachapoyas Para El Periodo de 2014-2018." Revista de Climatología 20: 15-28.

Rascón, J., W. Gosgot Angeles, L. Quiñones Huatangari, M. Oliva, y M. Á. Barrena Gurbillón. 2021a. "Dry and Wet Events in Andean Populations of Northern Peru: A Case Study of Chachapoyas, Peru." Frontiers in Environmental Science 9 (3): 1-13. DOI:10.3389/fenvs.2021.614438.

Rascón, J., F. Corroto, D. Leiva-Tafur, y O. A. Gamarra Torres. 2021b. "Variaciones Limnológicas Espaciotemporales de Un Lago Altoandino Tropical Al Norte de Perú.” Ecología Austral $31 \quad(2): \quad 343-56$. DOI:10.25260/ea.21.31.2.0.1200.

R Core Team. 2019. "R: A Language and Environment for Statistical Computing." R Foundation for Statistical Computing, Vienna, Austria. https://www.r-project.org/.

Tapia, Mario E, y Ana Maria Fries. 2007. Guia de Campo de Los Cultivos Andinos. Fao; AnpePerú. Lima (Perú): FAO y ANPE.

Zamora-Huamán, S. J. y F. L. Mejía-Risco. 2019. "Desarrollar Un Modelo de Predicción Mediante Espectroscopia En Infrarrojo Cercano (NIRS) Para La Determinación de Proteína Cruda En Subproductos de Arroz (Oriza Sativa)" Revista de Investigación Cientifica UNTRM: Ciencias Naturales e Ingeniería 2 (1): 60-65. 